\title{
Conmutadores ópticos no lineales e información cuántica a partir de nanocompositos metálicos anisotrópicos
}

\author{
Alicia Oliver* \\ JORGE ALEJANDRO REYES-ESQUEDA*
}

E uso de nuevos materiales del tamaño de una millonésima del espesor de un cabello, es decir de tamaño nanométrico, así como su respuesta óptica ultrarrápida a altas intensidades de la luz, promete revolucionar la comunicación entre los seres humanos mediante la creación de conmutadores ópticos no lineales y el manejo de la información a través de métodos cuánticos. En el Instituto de Física de la UNAM, estamos dirigiendo nuestros esfuerzos en esa dirección a través de la fabricación de nanopartículas metálicas deformadas y embebidas en una matriz dieléctrica, tal como el vidrio, obteniendo así nanocompositos metálicos anisotrópicos capaces de lograr tal revolución.
$\mathbf{L}$ a necesidad de mayor rapidez en la conmutación de señales es lo que ha permitido los grandes avances en la computación y en general en las telecomunicaciones, por ejemplo la implementación de señales de GPS (Global Positioning System, en Inglés) en teléfonos móviles. Sin embargo, la microelectrónica desde hace una década llegó a sus límites, tanto de confinamiento electrónico como económico. Esto ha significado el tener que dar paso a la optoelectrónica, pero aún así, como la conmutación más rápida se da cuando el proceso es completamente óptico, actualmente se busca que la fotónica reduzca las dimensiones de sus dispositivos para ser competitiva en tamaño con la microelectrónica integrada.

En este sentido, la conmutación óptica de señales es una aplicación que fue propuesta hace ya algunos años para realizar operaciones lógicas con luz, las cuales serían utilizadas en sistemas de procesamiento óptico de información. El principio de operación de estos sistemas está basado en la obtención de una interacción entre dos haces de luz, explotando la respuesta óptica no lineal del material en que se propagan (Figura 1). La

\footnotetext{
* Instituto de Física, Universidad Nacional Autónoma de México, Correspondencia: oliver@fisica. UNAM.MX; REYES@FISICA.UNAM.MX
}

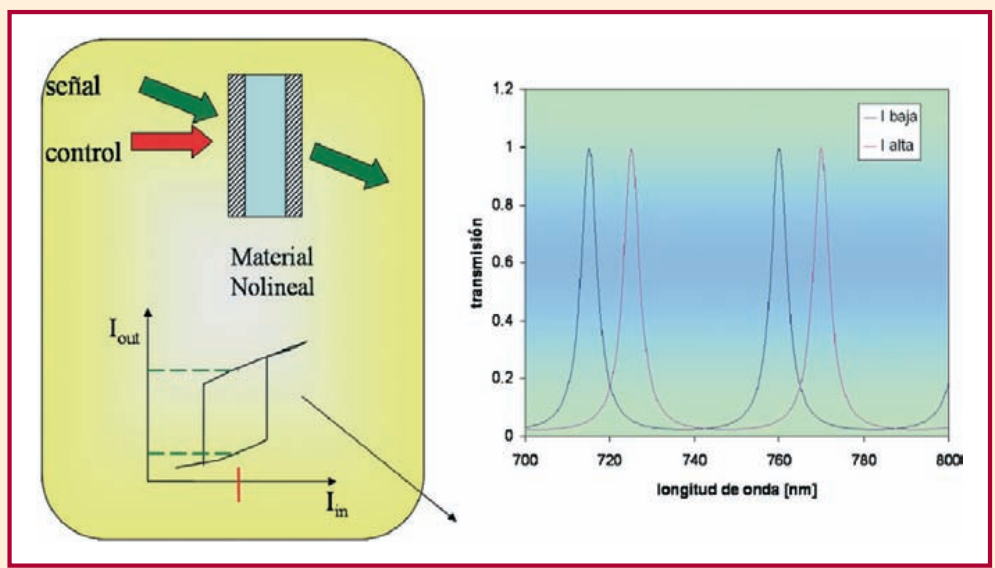

FIGURA 1. Conmutación óptica no lineal para producir 0’s y 1's con luz. 
idea era aprovechar la muy alta frecuencia de la luz, lo que en principio permitiría procesar información a tasas mucho más altas que los medios electrónicos convencionales.

Las dificultades técnicas encontradas en la implementación de estas compuertas, han impedido la fabricación y empleo de dispositivos prácticos. Estas dificultades tienen que ver en parte con la falta de materiales con las características idóneas, tales como una no linealidad óptica alta y un tiempo de respuesta ultracorto, y en parte, con la adopción de arquitecturas estándar óptimas. Por ejemplo, el tener una respuesta óptica no lineal significa utilizar altas intensidades de la luz, las cuales pueden a su vez dañar la estructura del material y, por tanto, reducir el tiempo de vida del dispositivo.

Se ha intentado revertir estas dificultades debido al gran auge de las telecomunicaciones ópticas en los últimos años y a la necesidad de eliminar los cuellos de botella originados por dispositivos de conversión óptico-eléctrico-óptico de los conmutadores y repetidores actuales en estos sistemas. En particular existe un gran interés en la implementación de funciones de conmutación de señales ópticas. Por ejemplo, la técnica de multiplexado óptico por división en el tiempo (отрм optical time division multiplexing, en inglés), requiere conmutadores ópticos con tiempos de respuesta ultrarrápidos (Figura 2).

Así mismo, la integración de dichos dispositivos de conmutación completamente ópticos en sistemas de telecomunicaciones, hace que la elección de dispositivos basados en guías de onda sean particularmente atractivos.

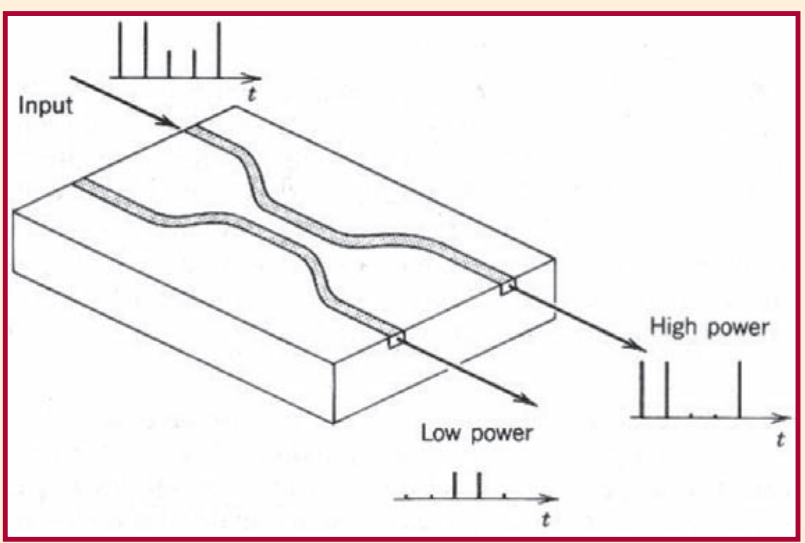

FIGURA 2. Conmutador óptica no lineal en guía de onda para técnica de multiplexado óptico por división en el tiempo.
También existe una nueva área de la información que tiene que ver con nuevos materiales con una respuesta óptica no lineal importante y ultrarrápida, la cual promete causar una nueva revolución tecnológica en las telecomunicaciones. Esta área es la de la información cuántica, donde se tiene una amplia gama de resultados que contradicen nuestra intuición. Así, por ejemplo, en una computadora cuántica sería posible, en principio, escribir un programa informático para resolver un problema y obtener la solución a él sin necesidad de ejecutar tal programa, aunque siempre sería necesario prender, al menos, tal computadora (Dowling, 2006; Hosten et al., 2006). 
Por todo lo anterior, se ha explorado y evaluado la respuesta óptica no lineal observada en toda una gama de materiales para su posible explotación en este tipo de nuevos dispositivos. Los materiales estudiados incluyen: semiconductores, polímeros, vidrios y nanoparticulas (NPs). En particular, NPs metálicas embebidas en matrices vítreas han mostrado tener coeficientes ópticos no lineales relativamente grandes con tiempos de respuesta ultrarrápidos.

La síntesis de NPs metálicas por implantación de iones proporciona un excelente método para la construcción de conmutadores ópticos, debido a que esta técnica produce guías de ondas en forma natural. El Instituto de Física de la UNAM cuenta con un acelerador de partículas que puede implantar una gran diversidad de iones en sólidos, entre ellos metales nobles. Debido a todo esto, nos hemos propuesto estudiar la implementación de dispositivos prototípicos de conmutación completamente óptica de señales, usando geometrías de guiado de ondas basados en nanopartículas metálicas en matrices vítreas, producidas por implantación iónica. De esta forma, queremos aprovechar la respuesta óptica no lineal ultrarrápida de las nanopartículas, combinada con la conveniencia de una arquitectura de guías de onda, para producir dispositivos con un potencial muy alto en aplicaciones de conmutación completamente óptica de señales. Esta arquitectura también está comenzando a mostrar grandes ventajas en la generación de puertos lógicos en información cuántica (Garay-Palmett. et al., 2007; Takesue et al., 2008).

Actualmente, el gran problema de la fotónica es que el guiado de las ondas electromagnéticas por las guías de onda convencionales ocupa espacios de micras, lo que hace que dichos dispositivos sean voluminosos, en contrapartida con la microelectrónica. Sin embargo, se está estudiando la posibilidad de transmitir la energía de una onda electromagnética a través de los modos de resonancia del plasmón de superficie de nanopartículas metálicas embebidas en un dieléctrico transparente, lo que permitiría el transporte de información por guías nanométricas, tanto del tipo clásica como cuántica (Lassiter et al., 2008, Reyes-Esqueda, sin fecha).

Debido a todo lo anterior, desde hace diez años, miembros del Grupo de Análisis y Modificación de Materiales con Aceleradores de Iones (GAMMAI) del IfunAm, Dr. Juan Carlos Cheang Wong, Dr. Luis Rodríguez, Dr. Alejandro Crespo, Dra. Alejandra López y Dra. Alicia Oliver, han estudiado, usando el Acelerador "Marcos Mazari" del IFUnAM [Figura 3], la nucleación de nanopartículas metálicas y semiconductoras en dióxido de silicio $\left(\mathrm{SiO}_{2}\right)$, por medio de la implantación de iones a energías del orden de megaelectronvolts, MeV (Peña et al., 2007A y 2006; Roiz et al., 2004; Duong et al., 2003; Barthou et al., 2003; Oliver et al., 2002, 2000 y 1998; Cheang-Wong et al., 


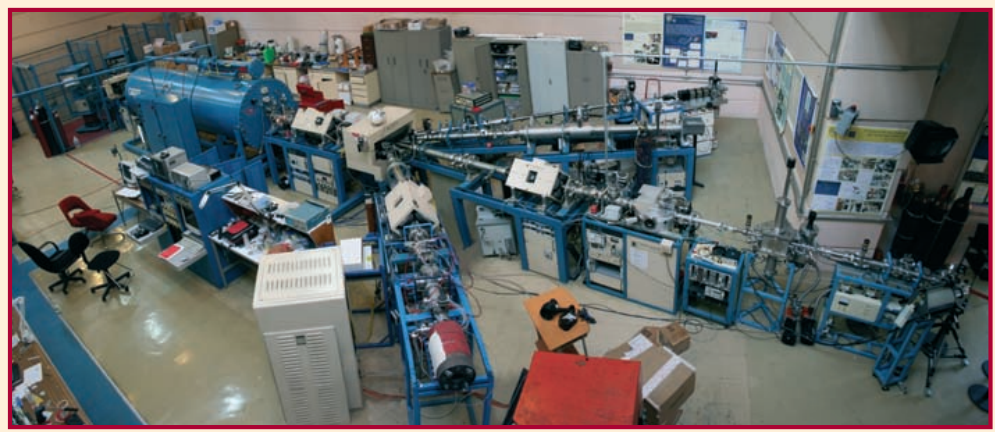

FIGURA 3. Acelerador "Marcos Mazari" del IfUnAm.

2001AA, 2001B y 2000; y Pal et al., 2000). Gracias a esta experiencia, se ha podido reducir el ancho de la distribución de tamaños de las nanopartículas por medio del control de los parámetros de la implantación de iones y de recocidos a diferentes temperaturas en diferentes atmósferas. Recientemente, se publicó en la literatura que la sílice se podía deformar por medio de la irradiación de iones con energías del orden de decenas de MeV. El grupo experimentó y pudo confirmar este hecho irradiando partículas de $\mathrm{SiO}_{2}$ con iones a estas energías (Cheang-Wong et al., 2006 y 2007). Con base en esta experiencia, se propuso hacer experimentos con el fin de deformar nanopartículas de Plata (Ag) en $\mathrm{SiO}_{2}$, metal en el cual no se había observado deformación bajo las condiciones mencionadas, suponiendo, acertadamente, que el que otros grupos no pudieran observar la deformación en Ag por microscopía electrónica de transmisión (TEM), era debido a la alta movilidad de la Ag en la sílice, y que la nanopartícula de Ag más la sílice circundante, al estar en interacción con el haz de electrones, regresaba a su configuración de simetría esférica, perdiendo su estructura alargada. Por lo tanto, propusimos como método de análisis a la absorción óptica como método complementario al de la microscopía electrónica. Los resultados obtenidos mostraron que efectivamente se podía cambiar la forma de nanopartículas de Ag en $\mathrm{SiO}_{2}$ por medio de irradiación con iones de decenas de $\mathrm{MeV}$, transformándolas de una forma cuasiesférica a una alargada en forma controlada (Figuras 4 y 5). Este resultado (Oliver et al., 2006), el primero para partículas de Ag, abre una gran posibilidad de manipular el plasmón de superficie en forma controlada. El hecho de que se tratara de nanopartículas de Ag es importante, ya que la banda de absorción de la resonancia del plasmón de superficie de este metal es particularmente estrecha, comparada con la de oro $(\mathrm{Au})$ y cobre $(\mathrm{Cu})$, de tal manera que esta banda está muy bien definida y es intensa, permitiendo hacer un buen seguimiento del plasmón de superficie, y dando información sobre la posibilidad de transportar con 


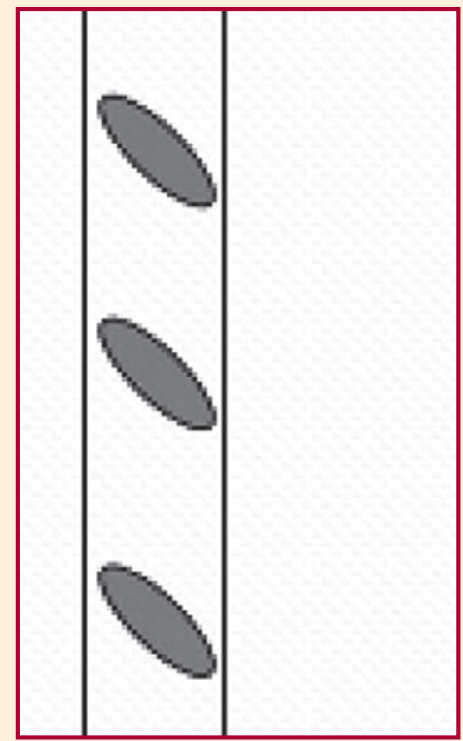

FIGURA 4. Esquema del arreglo de nanopartículas metálicas alargadas y orientadas en la misma dirección dentro de una matriz vítrea. eficiencia energía electromagnética (Van Hulst, 2007; De Waele et al., 2007). Este hecho es de suma importancia ya que, de poderse transportar la energía de una onda electromagnética a través de los modos de la resonancia del plasmón de superficie de nanopartículas metálicas, y poder recuperarla otra vez como onda electromagnética, permitirá reducir el tamaño de micras para la transmisión de señales en el infrarrojo para telecomunicaciones (guías de onda convencionales), a dimensiones de decenas de nanómetros, a través de un cierto arreglo de nanopartículas metálicas. Esta aplicación tiene todavía muchos problemas técnicos difíciles de resolver, sin embargo, existen algunos grupos en el mundo trabajando en ello.

Igualmente, este arreglo de nanopartículas metálicas alargadas y orientadas todas en la misma dirección le confiere a dicho arreglo contenido en una matriz de sílice una respuesta óptica conocida como birrefringencia, la cual consiste en mostrar una anisotropía del índice de refracción, es decir diferentes índices para cada eje de simetría del material. Esta birrefringencia, aunada a la respuesta óptica no lineal del material, permitiría, en principio, generar fotones enredados en su estado de polarización a partir de nanopartículas metálicas anisotrópicas. Esto es, fotones que reaccionarían de manera instantánea (Rudolph et al., 2008) a la manipulación del estado de polarización de alguno de ellos.

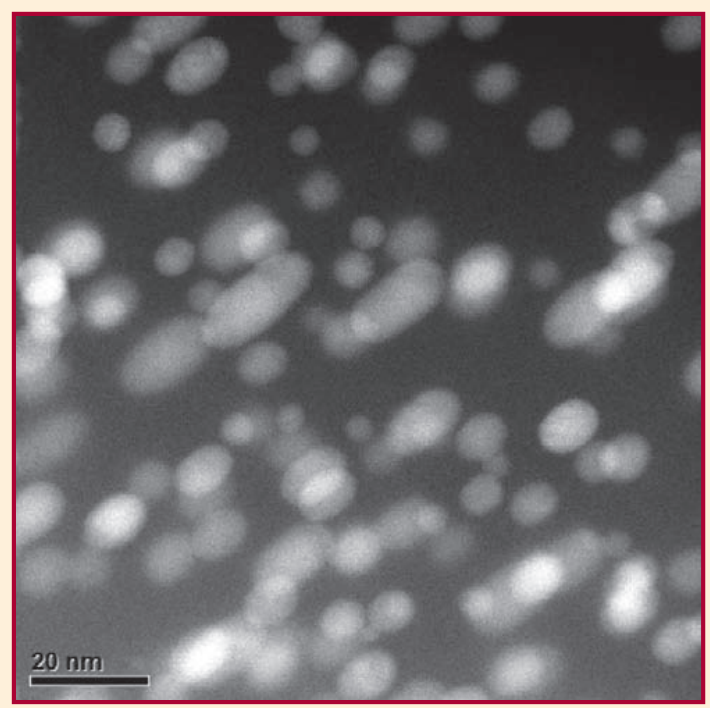

FIGURA 5. Micrografía electrónica por contraste $Z$ del esquema anterior.

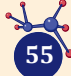


La formación de guías de onda por implantación ya es un hecho, por lo tanto nos hemos enfocado por el momento en construir guías de onda con nanopartículas de $\mathrm{Ag}$ y $\mathrm{Au}$ que tengan una alta respuesta óptica no lineal. La respuesta óptica no lineal en los dieléctricos con nanopartículas metálicas se debe mayormente a procesos de tercer orden, los cuales se pueden describir como la modificación, mediante un haz intenso de luz, del índice de refracción del material y/o su absorción óptica. La modificación del índice de refracción se conoce como el efecto Kerr, el cual es el más importante para las aplicaciones en todos los dispositivos de conmutación óptica. Es por esto que el grupo GAMmaI sumó sus esfuerzos a los del Dr. Alejandro Reyes del Departamento de Estado Sólido del mismo IFunAm, asesorados por el Dr. Raúl Rangel Rojo del Centro de Investigación Científica y de Educación Superior de Ensenada, Baja California (CICESE), para entender y mejorar la respuesta óptica no lineal de este tipo de materiales (Torres-Torres et al., 2007 y 2008; Fandiño et al., 2008; Reyes-Esqueda et al., 2008; Rodríguez-Iglesias et al., 2008; Ranjel-Rojo et al., 2008; Peña et al., 2007B), y del Dr. Heriberto Márquez del mismo centro, en la búsqueda de las aplicaciones mencionadas antes (Figura 6).

Esto pone de relieve a los estudios de la banda de absorción del plasmón de superficie para proporcionar información sobre la respuesta óptica no lineal y la calidad de transmisión en las guías de onda, así como sobre las condiciones de la nucleación de las nanopartículas metálicas que presenten fuertes acoplamientos que propaguen los modos del plasmón de superfi-

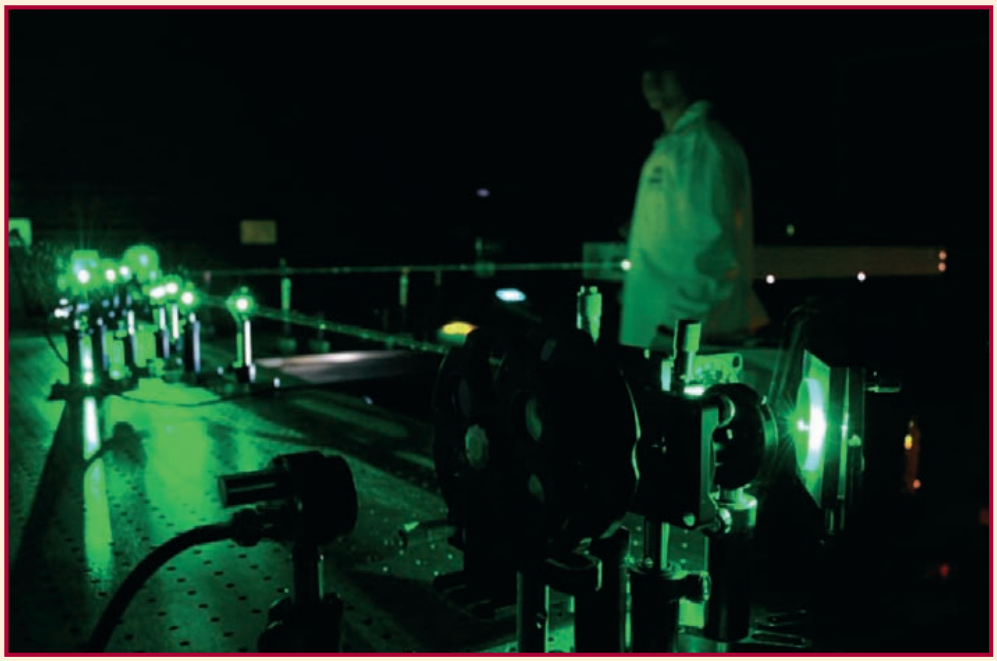

FIGURA 6. Laboratorio de óptica no lineal del IFUnAm. 


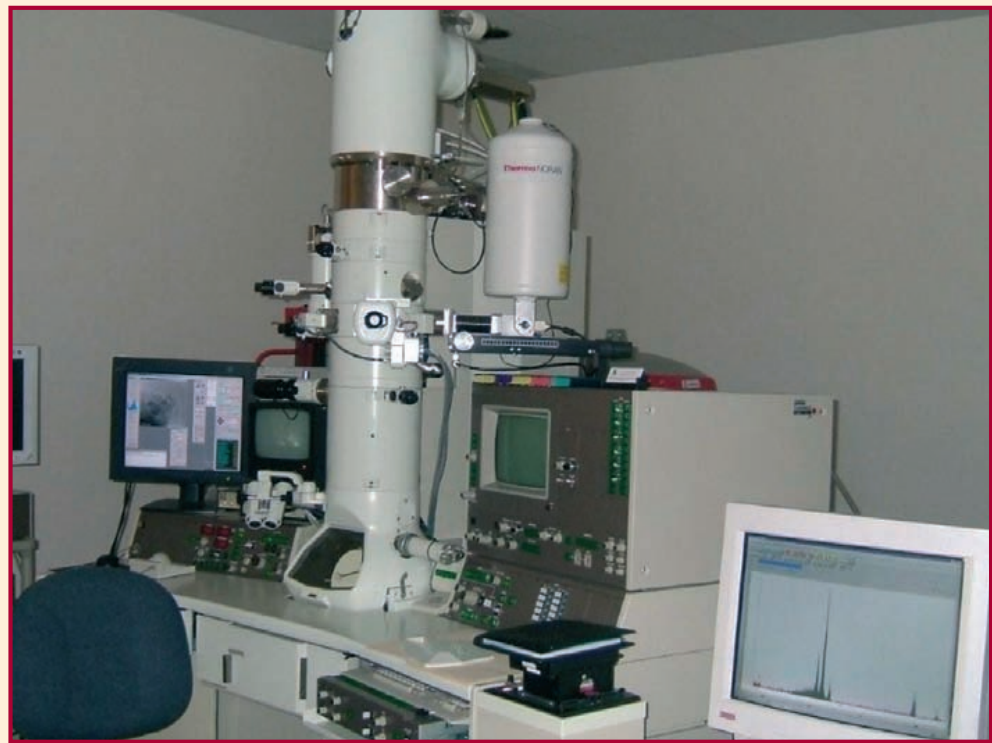

FIGURA 7. MIcroscopio electrónico de transmisión de emisión de campo del ifunam.

cie. Estos estudios son muy importantes para poder conseguir la meta de guiar la información electromagnética por guías nanométricas (Inouye et al., 2000).

Sin embargo, también ha sido necesario retomar el estudio de estos materiales mediante microscopía electrónica, ya que las propiedades ópticas

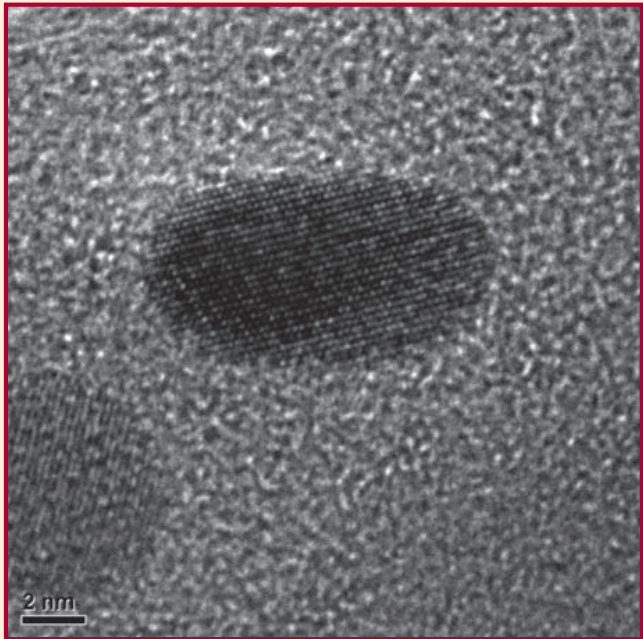

FIGURA 8. Microscopía electrónica de alta resolución de NPs de Au deformadas. de las nanopartículas dependen del tamaño y forma de éstas, y dadas las prometedoras perspectivas que tienen los materiales dieléctricos con nanopartículas metálicas para aplicaciones, ya sean cuasiesféricas o alargadas, el grupo GAMmAI junto con el Dr. Jesús Arenas del Departamento de Materia Condensada, también del IFUnAm y el Dr. G Kellerman de Brasil, se han avocado a hacer todo un estudio de su distribución de tamaños, de su distribución de formas, y de su composición, utilizando microscopía electrónica Peña et al., 2007A; Cheang-Wong et al., 2007B) (figuras 7 y 8) y dispersión de rayos-x a ángulo pequeño con incidencia rasante utilizando radiación de sincrotrón (GISAXS) (Rodríguez-Iglesias et al., 2008).

En conclusión, los estudios llevados a cabo en el Instituto de Física de la UNAM, así como por supuesto, en otras partes del mundo, tarde o temprano darán resultados tecnológicos que revolucionarán el manejo de la información que conocemos ahora. Si de hecho, 
el uso de los teléfonos portátiles y las computadoras ha causado ya una revolución en la comunicación entres los seres humanos, la nanotecnología y la información cuántica prometen revolucionarlo aún más, llevándonos a límites que ni sólo la ciencia ficción se ha atrevido a explorar. Y nuestro sentir es que eso sucederá muy pronto.

\section{REFERENCIAS}

Barthou, C., Duong, P. H., Oliver, A., Cheang-Wong, J. C., Rodríguez-Fernández, L., Crespo-Sosa, A., Itoh, T., y Lavallard, P., (2003). "Silicon nanocrystals and defects produced by silicon and silicon-and-gold implantation in silica". Journal of Applied Physics. 93, 10110.

Cheang-Wong, J. C., Morales, U., Reséndiz, E., Oliver, A., y Rodríguez-Fernández, L., (2007 A). "Deformation of colloidal silica particles using MeV Si ionirradiation”. Journal of Non-Crystalline Solids. 353, 1925.

, Oliver, A., Rodríguez-Fernández, L., Arenas-Alatorre, J., Peña, O., y Crespo-Sosa, A., (2007 B). "Optical absorption and HRTEM characterization of metallic nanoparticles produced by MeV Ion implantation". Revista Mexicana de Física S 53, 49.

, Morales, U., Oliver, A., Rodríguez-Fernández, L., y Rickards, J., (2006). "MeV ion beam deformation of colloidal silica particles". Nuclear Instruments and Methods in Physics Research B. 242, 452.

Oliver, A., Roiz, J., Hernández, J. M., Rodríguez-Fernández, L., y Crespo, A., (2001 A). "Optical Absorption and Emission Study of $2 \mathrm{MeV} \mathrm{Cu}-$ Implanted Silica Glass". Nuclear Instruments and Methods in Physics Research B. 175-177, 495.

, Oliver, A., Roiz, J., Hernández, J. M., Rodríguez-Fernández, L., Morales, J. G., Crespo, A., (2001 B). “Optical Properties of Ir2+-Implanted Silica Glass”. Nuclear Instruments and Methods in Physics Research B. 175-177, 490.

, Oliver, A., Roiz, J., Rodríguez-Fernandez, L., Hernández, J. M., CrespoSosa, A., (2001 C). "Relationship between the Ag depth profiles and the nanoparticle formation in Ag-implanted silica". Journal of Physics: Condensed Matter. 13, 10207.

, Oliver, A., Crespo, A., Hernández, J. M., Muñoz, E., y Espejel-Morales, R., (2000). "Dependence of the optical properties on the ion implanted depth profiles in fused quartz after a sequential implantation with $\mathrm{Si}$ and $\mathrm{Au}$ ions". Nuclear Instruments and Methods in Physics Research B. 161-163, 1058.

De Waele, R., Koenderink, A. F., y Polman, A., (2007).“Tunable nanoescale localization of energy on plasmon particle arrays". Nano Letters. 7, 2004.

Dowling, P (2006). “To compute or not to compute?" Nature 439, 919-920.

Duong, P. H., Lavallard, P., Oliver, A., e Itoh, T., (2003) “Temperature dependence of photoluminescence from localized states of silicon nanocrystals in silicon-implanted quartz". Physica Status Solidi C. Vol. 0, No. 4: 1271-1274.

Fandiño, J., García-Sánchez, M. F., Santana, G., Crespo, A., Alonso, J. C., y Oliver, A., (2008). "Correlation between microstructure of plasma-modified gold 
nanoclusters and their optical Properties". Superlattices and Microstructures. $43,454$.

Garay-Palmett, K., McGuinness, H. J., Cohen, O., Lundeen, J. S., Rangel-Rojo, R., U’Ren, A. B., Raymer, M. G., McKinstrie, C.J., Radic, S., y Walmsley, I. A., (2007). "Photon pair-state preparation with tailored spectral properties by spontaneous four-wave mixing in photonic-crystal fiber". Optics Express. Vol. 15, No. 22: 14870.

Hosten, O., Rakher, M. T., Barreiro, J. T., Peters, N. A., y Kwiat, P. G (2006). “Counterfactual quantum computation through quantum interrogation". Nature 439, 949-952.

Inouye, H., Tanaka, K., Tanahashi, I., Hattori, T., y Nakatsuka, H., (2000). “Ultrafast Optical Switching in a Silver Nanoparticle System". Japanese Journal of Applied Physics. 39, 5132.

Lassiter,J. B., Aizpurua, J., Hernández, L. I., Brandl, D. W., Romero, I., Lal, S., Hafner, J. H., Nordlander, P., y Halas, N. J., (2008). “Close encounters between two nanoshells". Nanoletters No. 8: 1212.

Oliver, A., Reyes-Esqueda, J. A., Cheang-Wong, J. C., Román-Velázquez, C. E., Crespo-Sosa, A., Rodríguez-Fernández, L., Seman, J. A., Noguez, C., (2006). "Controlled anisotropic deformation of Ag nonoparticles by Si ion irradiation". Physical Review B. 74, 245425.

, Cheang-Wong, J. C., Roiz, J., Rodríguez-Fernández, L., Hernández, J. M., Crespo-Sosa, A., y Muñoz, E., (2002). "Metallic nanoparticle formation in ion-implanted silica after thermal annealing in reducing or oxiding atmosferes". Nuclear Instruments and Methods in Physics Research B. 191, 333.

, Cheang, J. C., Crespo, A., Rodríguez-Fernández, L., Hernández, J. M., Muñoz, E., y Espejel-Morales, R., (2000) “E” and B2 Center Production in Amorphous quartz by MeV Si and Au Ion Implantation". Materials Science and Engineering B. 78, 32.

, Cheang-Wong, J. C., Crespo, A., Hernández, J. M., Solís, C., Muñoz, E., Espejel-Morales, R., y Siejka, J., (1998). "Study of the optical properties of fused quartz after a sequential implantation with Si and Au ions". Applied Physics Letters. 73, 1574.

Pal, U., Bautista-Hernández, A., Rodríguez-Fernández, L., y Cheang-Wong, J. C., (2000). "Effect of Thermal Annealing on the Optical Properties of HighEnergy Cu Implanted Silica Glass". Journal of Non-Crystalline Solids. 275, 65.

Peña, O., Cheang-Wong, J. C., Rodriguez-Fernández, L., Arenas-Alatorre, J., Crespo-Sosa, A., Rodríguez-Iglesias, V., Oliver, A., (2007 A). “Metal and metal oxide nanoparticles produced by ion implantation in silica: A microstructural study using HRTEM". Nuclear Instruments and Methods B. Vol. 257, No. 99.

, Rodríguez-Fernández, L., Roiz, J., Cheang-Wong, J. C., Arenas-Alatorre, J., Crespo-Sosa, A., y Oliver, A., (2007 B). "Average size of Ag nanoclusters insilica determined by optical light absorption measurements". Revista Mexicana de Física. S 53, 62.

, Rodríguez-Fernández, L., Cheang-Wong, J. C., Santiago, P., Crespo-Sosa, A., Muñoz, E., y Oliver, A., (2006). "Characterization of nanocluster forma- 
tion in Cu-implanted silica: Influence of the annealing atmosphere and the ion fluence". Journal of Non-Crystaline Solids. 352, 349.

Rangel-Rojo, R., McCarthy, J., Bookey, H. T., Kar, A. K., Rodriguez-Fernandez, L., Cheang-Wong, J. C., Crespo-Sosa, A., Lopez-Suarez, A., Oliver, A., Rodriguez-Iglesias, V., y Silva-Pereyra, H. G., (2008). "Anisotropy in the nonlinear absorption of elongated silver nanoparticles in a silica matrix, probed by femtosecond pulses". Trabajo sometido a: Conference on Lasers and Electro-Optics - CLEO.

Reyes-Esqueda, J. A., Torres-Torres, C., Cheang-Wong, J. C., Crespo-Sosa, A., Rodriguez-Fernández, L., Noguez, C., y Oliver, A., (2008). "Large optical birefringence by anisotropic silver nanocomposites”. Optics Express. 16: 710.

Reyes-Esqueda, J. A (sin fecha).“Información cuántica a partir de nanocompositos metálicos anisotrópicos". Proyecto de Investigación CONACYT. IFUNAM. México.

Rodriguez-Iglesias, V., Silva-Pereyra, H. G., Cheang-Wong, J. C., Reyes-Esqueda, J. A., Fernández-Fernández, L., Crespo-Sosa, A., Kellerman, G., y Oliver, A., (2008). "MeV Si ion iradiation effects on tha optical absorption properties of metallic nanoparticles embedded in silica". Nuclear Instruments and Methods B. 266, 3138.

Roiz, J., Oliver, A., Muñoz, E., Rodríguez-Fernández, L., Hernández, J. M., y Cheang-Wong, J.C., (2004) "Modification of the optical properties of Agimplanted silica by annealing in two different atmospheres". Journal of Applied Physics. 95, 1783.

Rudolph, T. G., (2008). “The speed of instantly”. Nature. 454, 831.

Salart, D., Baas, A., Branciard, C., Gisin, N., y Zbinden, H., (2008). Nature. 454, 861.

Takesue, H., Fukuda, H., Tsuchizawa, T., Watanabe, T., Yamada, K., Tokura, Y., e Itabashi, S., (2008). "Generation of polarization entangled photon pairs using silicon wire waveguide”. Optics Express. Vol. 16, No. 8: 5721.

Torres-Torres, C., Reyes-Esqueda, J. A., Cheang-Wong, J. C., Crespo-Sosa, A., Rodríguez-Fernández, L., y Oliver, A., (2008). “Optical third-order nonlinearity by nanosecond and picosecond pulses in $\mathrm{Cu}$ nanoparticles in ion-implanted silica". Journal of Applied Physics. 104, 014306.

, Khomenko, A. V., Cheang-Wong, J. C., Rodríguez-Fernández, L., CrespoSosa, A., y Oliver, A., (2007). "Absorptive and refractive nonlinearities by four-wave mixing for Au nanoparticles in ion-implanted silica". Optics Express. 15, 9248.

, López-Suárez, A., Tamayo-Rivera, L., Rangel-Rojo, R., Crespo-Sosa, A., Oliver, A., (sin fecha). "Thermo-optic effect and optical third order nonlinearity in nc-Si embedded in a silicon-nitride film". Optic Express. Aceptado.

Van Hulst, N. F., (2007).“Light in chains”. Nature. Vol. 448, No. 141. 